\title{
PERIODIC POINTS FOR HOMEOMORPHISMS OF HEREDITARILY DECOMPOSABLE CHAINABLE CONTINUA
}

\author{
W. T. INGRAM
}

(Communicated by Dennis Burke)

\begin{abstract}
In this paper it is shown that homeomorphisms of hereditarily decomposable chainable continua cannot have periodic points whose periods are not powers of two. Examples show that for each power of two there is a hereditarily decomposable chainable continuum and a homeomorphism of it which has a periodic point of period that power of two.
\end{abstract}

\section{INTRODUCTION}

In a paper in Fundamenta Mathematicae in 1979, Michel Smith and Sam Young showed that hereditarily decomposable chainable continua do not have homeomorphisms whose $n$th composite is the identity except for $n=1$ and $n=2$ [5]. In this paper we show that if $h$ is a homeomorphism of a hereditarily decomposable chainable continuum then $h$ does not have a periodic point of prime period $n$ unless $n$ is a power of 2 . We give two arguments for this result. One proof uses the recent result of Piotr Minc and W. R. R. Transue [4] that Sarkovskii's Theorem holds for hereditarily decomposable chainable continua. The other proof uses an extension (see Theorem 5 of section 3) of a result of the author [2, Theorem 3]. In the process of proving the theorem this second way we obtain some theorems (e.g., Theorem 4) which may be useful in their own right.

By a continuum we mean a compact, connected metric space. By a mapping we mean a continuous function. If $f$ is a mapping of a continuum into itself, a point $x$ is said to be a periodic point for $f$ provided there is a positive integer $n$ such that $f^{n}(x)=x$. If $x$ is a periodic point for $f$, we say that $x$ has prime period $n$ (or simply, period $n$ ) if $n$ is the least positive integer $i$ such that $f^{i}(x)=x$.

Received by the editors June 27, 1988 and, in revised forms, December 1, 1988 and February 27, 1989.

1980 Mathematics Subject Classification (1985 Revision). Primary 54F20, 54H20; Secondary 54F62, 54H25, 54F55.

Key words and phrases. Periodic point, atriodic, unicoherent, chainable continuum, indecomposable continuum, inverse limit. 
A continuum $M$ is a triod if it contains a subcontinuum $C$ such that $M-C$ his at least three components. A continuum is atriodic if it contains no triod. The statement that the continuum $M$ is unicoherent means if $M$ is the union of two subcontinua $H$ and $K$ then the common part of $H$ and $K$ is connected. A continuum is called hereditarily unicoherent provided each of its subcontinua is unicoherent. A continuum is decomposable if it is the union of two of its proper subcontinua and indecomposable otherwise. A continuum is hereditarily decomposable if each of its nondegenerate subcontinua is decomposable.

If $X_{1}, X_{2}, X_{3}, \ldots$ is a sequence of spaces and $f_{1}, f_{2}, f_{3}, \ldots$ is a sequence of mappings such that, for each positive integer $i, f_{i}: X_{i+1} \rightarrow X_{i}$, then by the inverse limit of the inverse system $\left\{X_{i}, f_{i}\right\}$ is meant the subset of the product of the spaces $X_{1}, X_{2}, \ldots$ to which the point $\left(x_{1}, x_{2}, \ldots\right)$ belongs if and only if $f_{i}\left(x_{i+1}\right)=x_{i}$. A continuum is chainable if it is homeomorphic to an inverse limit of $\left\{X_{i}, f_{i}\right\}$ where, for each $i, X_{i}=[0,1]$. Suppose $f$ is a mapping of a continuum $M$ into itself and, for each positive integer $i, X_{i}=M$ and $f_{i}=f$. We denote by $\lim \{M, f\}$ the inverse limit of the inverse system $\left\{X_{i}, f_{i}\right\}$.

If $x$ is a periodic point for a mapping $f$ of period $n$, the orbit of $x$, denoted $O(x, f)$ (or simply, $(O(x))$, is $\left\{x, f(x), \ldots, f^{n-1}(x)\right\}$.

\section{USING THE SARKOVSKII-TYPE THEOREM}

Recently, Minc and Transue showed that Sarkovskii's Theorem holds for hereditarily decomposable chainable continua. Consider the Sarkovskii order on the positive integers: $3 \triangleright 5 \triangleright 7 \triangleright \cdots \triangleright 2 \cdot 3 \triangleright 2 \cdot 5 \triangleright 2 \cdot 7 \triangleright \cdots \triangleright 2^{2} \cdot 3 \triangleright$ $2^{3} \cdot 3 \triangleright \cdots \triangleright \cdots \triangleright 2^{3} \triangleright 2^{2} \triangleright 2 \triangleright 1$. Suppose $f$ is a mapping of the hereditarily decomposable chainable continuum $M$ into $M$ and $f$ has a periodic point of period $m$. If $m \triangleright k$, then $f$ has a periodic point of period $k$.

Theorem 1. If $h$ is a homeomorphism of the hereditarily decomposable chainable continuum $M$ and $x$ is a periodic point for $h$ of period $n$ then there is a nonnegative integer $j$ such that $n=2^{j}$.

Proof. Suppose $M$ is a hereditarily decomposable chainable continuum and $h$ is a homeomorphism of $M$ onto $M$ with a periodic point of period $n=k \cdot 2^{j}$ where $k$ is odd and greater than 1 . Then $x$ is a periodic point of period $k$ for $H=h^{2^{j}}$. Since $k$ is odd and greater than 1 , by the theorem of Minc and Transue, $H$ has a periodic point of period 6 . Thus $H^{2}$ has a periodic point of period 3. Since $M$ is atriodic and hereditarily unicoherent, by [2, Theorem 3], $\lim \left\{M, H^{2}\right\}$ contains an indecomposable continuum. However, since $M$ is homeomorphic to $\lim \left\{M, H^{2}\right\}$, this involves a contradiction.

\section{ATRIODIC AND hEREDITARILY UNICOHERENT CONTINUA}

In the proof presented in $\S 2$, the Sarkovskii-type theorem is used only to show that $H$ has a periodic point of period 6 because $H$ has a periodic point of odd period. One can get around the need for the Minc and Transue Theorem by 
generalizing [2, Theorem 3]. This is the purpose of this section (see Theorem 5).

Theorem 2. The continuum $M$ is atriodic and hereditarily unicoherent if and only if it is true that if $A, B$ and $C$ are three subcontinua of $M$ and $x, y$ and $z$ are three points such that $x$ is in $A$ and $B, y$ is in $B$ and $C$ and $z$ is in $C$ and $A$, then $\{x, y, z\}$ is a subset of one of $A, B$ and $C$.

Proof. Suppose $A, B$ and $C$ are three subcontinua of the atriodic and hereditarily unicoherent continuum $M$ and $x, y$ and $z$ are points such that $x$ is in $A$ and $B, y$ is in $B$ and $C$ and $z$ is in $A$ and $C$. Then the common part of $A$ and $(B \cup C)$ is a continuum. But, $A \cap(B \cup C)=(A \cap B) \cup(A \cap C)$, so there is a point which belongs to all three of $A, B$ and $C$. Thus there is a point common to $A \cap B, B \cap C$ and $A \cap C$. Since $M$ is atriodic, by a theorem of Sorgenfrey [6], one of the three is a subset of the union of the other two. If $A \cap B$ is a subset of $(B \cap C) \cup(A \cap C)=C \cap(A \cup B)$, then $\{x, y, z\}$ is a subset of $C$. A similar argument yields the proper conclusion in each of the other two cases.

On the other hand, suppose $M$ contains a triod, $T=H \cup K \cup L$ such that the common part of each two is the common part of all three and is a proper subcontinuum of each one of them. There exist points $x, y$ and $z$ such that $x$ is a point of $H$ which is not in $K \cup L, y$ is a point of $K$ which is not in $H \cup L$ and $z$ is a point of $L$ which is not in $H \cup K$. Then $A=H \cup L$, $B=K \cup H$ and $C=L \cup K$ have the property that $x$ is in $A$ and $B$ but not $C, y$ is in $B$ and $C$ but not $A$ and $z$ is in $A$ and $C$ but not $B$.

Now, suppose $M$ contains two continua $H$ and $K$ whose common part is not connected. Choose $z$ in $H \cap K$ and let $L$ be a component of $H \cap K$ which does not contain $z$. Let $H_{1}$ be a subcontinuum of $H$ which does not contain $z$ but contains $L$ and contains a point $x$ of $H$ which is not in $K$. Let $K_{1}$ be a subcontinuum of $K$ which does not contain $z$ but contains $L$ and contains a point $y$ of $K$ which is not in $H$. Then $A=H, B=H_{1} \cup K_{1}$ and $C=K$ have the property that $x$ is in $A$ and $B$ but not $C, y$ is in $B$ and $C$ but not $A$ and $z$ is in $A$ and $C$ but not $B$.

Theorem 3. Suppose $x$ is a periodic point for $f$ of period $n$ and $k$ is an integer less than $n$ such that $k$ and $n$ are relatively prime. Then $O(x, f)=O\left(x, f^{k}\right)$.

Corollary. If $x$ is a periodic point for $f$ of period $n$ and $n$ is odd, then $O(x, f)=O\left(x, f^{2}\right)$.

Remark. A simple example shows that the Corollary does not necessarily hold for $n$ even. Let $f$ be defined on $[0,1]$ by $f(0)=2 / 3, f(1 / 3)=1, f(2 / 3)=$ $1 / 3, f(1)=0$ and $f$ is linear on each of the intervals $[0,1 / 3],[1 / 3,2 / 3]$ and $[2 / 3,1]$. Then 0 is a periodic of period 4 and $O(0, f)=\{0,1 / 3,2 / 3,1\}$ while $O\left(0, f^{2}\right)=\{0,2 / 3\}$. This example also shows that, in Theorem 4 , the hypothesis that " $H$ is a subcontinuum of $M$ containing $x$ and $f(x)$ " cannot be replaced by " $H$ is a subcontinuum of $M$ containing $x$ and $f^{2}(x)$ ". 
Theorem 4. If $M$ is atriodic and hereditarily unicoherent, $f$ is a mapping of $M$ into $M, x$ is a periodic point for $f$ of period $n$ and $H$ is a subcontinuum of $M$ containing $x$ and $f(x)$, then there is a positive integer $m$ such that $f^{m}(H)$ contains $O(x)$.

Proof. If $x$ is a fixed point for $f$ or a periodic point of period 2 for $f$, the conclusion clearly holds. So, suppose $x$ is a periodic point for $f$ of prime period $n>2$ and $H$ is a subcontinuum of $M$ containing $x$ and $f(x)$. Each iterate of $H$ (i.e. $\left.f^{i}(H)\right)$ contains at least two points of $O(x)$. If the conclusion is not true, then there is an integer $k, 1<k<n$, such that some iterate of $H$ contains $k$ points of $O(x)$ but no iterate of $H$ contains more than $k$ points of $O(x)$. Suppose $i$ is an integer such that $f^{j}(H)$ contains $k$ points of $O(x)$. Note that if $j>i, f^{j}(H)$ contains $k$ points of $O(x)$. Since $k<n$, there is a positive integer $j \geq i$ such that $x$ and $f(x)$ both lie in $f^{j}(H)$ but $f^{n-1}(x)$ is not in $f^{j}(H)$. To see that this is true, consider an iterate $I=f^{s}(H)$ with $s>i$ and $s=t \cdot n$ for some positive integer $t$. Then $I$ contains $x$ and $f(x)$. Since $I$ contains only $k$ members of $O(x)$, there is a positive integer $m$ less than $n$ such that $f^{m}(x)$ is not in $I$. Let $r$ be the greatest integer $m$ less than $n$ such that $f^{m}(x)$ is not in $I$. Let $j=s+n-(r+1)$.

There is a positive integer $p, p<n-1$, such that $f^{p}(x)$ is in $f^{j}(H)$ but if $p<t \leq n-1, f^{t}(x)$ is not in $f^{j}(H)$. Let $A=f^{j}(H), B=f(A)$ and $C=f(B) \cup \cdots \cup f^{n-p-1}(B)$. Note that $x$ is in both $A$ and $C$ but not in $B$ while $f(x)$ is in both $A$ and $B$ but not in $C$. To see that $f(x)$ is not in $C$ observe that if $f(x)$ is in $f^{m}(B)$ for some $m, 1 \leq m \leq n-p-1$, then $f(x)$ is in $f^{m+1}(A)$. From this it follows that $f^{n-m}(x)$ is in $f^{n}(A)$. Since, by the choice of $k, f^{n}(A)=A$ and $p+1 \leq n-m \leq n$, we have a contradiction to the choice of $p$. Finally, let $q$ be the least positive integer $i$ such that $f^{i}(x)$ is not in $A$. Then, $2 \leq q \leq p+1$, so $f^{q-1}(x)$ and $f^{q-2}(x)$ are both in $A$. Consequently, $f^{q}(x)$ is in both $B$ and $C$ but not $A$. This contradicts Theorem 2 and the proof is complete.

Remark. The proof of Theorem 5 uses a theorem of Kuykendall [3, Theorem 2 , p. 267]. Kuykendall proved that $\lim \left\{X_{i}, f_{i}\right\}$ is indecomposable if and only if for each positive integer $i$ and each positive number $e$ there exist a positive integer $n>i$ and three points of $X_{n}$ such that if $L$ is a subcontinuum of $X_{n}$ containing two of them then $d\left(x, f_{i} \circ f_{i+1} \circ \cdots \circ f_{n-1}(L)\right)<e$ for each $x$ in $X_{i}$.

Theorem 5. If $M$ is an atriodic and hereditarily unicoherent continuum and $f$ is a mapping of $M$ into $M$ with a periodic point of period $n$ where $n$ is odd and greater than 1, then $\lim \{M, f\}$ contains an indecomposable continuum.

Proof. Let $K$ be irreducible from $x$ to $f(x)$ and let $H=\operatorname{cl}\left(\bigcup_{i>0} f^{i}(K)\right)$. We show $\lim \{H, f \mid H\}$ is indecomposable by showing that the hypothesis of Kuykendall's Theorem [3] is satisfied. The three points we need are $x, f(x)$ 
and $f^{2}(x)$. If $L$ is a subcontinuum of $H$ containing two of these then by Theorems 3 and 4 there is a positive integer $m$ such that $f^{m}(L)$ contains $O(x)$. Thus, $\operatorname{cl}\left(\bigcup f^{i}(L)\right)=H$. By Kuykendall's Theorem, $\lim \{H, f \mid H\}$ is indecomposable.

We now give a second proof of Theorem 1:

Proof. Suppose $M$ is a hereditarily decomposable chainable continuum and $h$ is a homeomorphism of $M$ onto $M$ with a periodic point of period $n=$ $k \cdot 2^{j}$, where $k$ is odd and greater than 1. Then, $H=h^{2^{j}}$ has a periodic point of period $k$. By Theorem $5, \lim \{M, H\}$ contains an indecomposable continuum. However, $M$ is homeomorphic to $\lim \{M, H\}$ and this involves a contradiction.

Remark. Although the theorems of this section are for atriodic and hereditarily unicoherent continua and there are many examples of such continua which are not chainable, the present application of these results to give this second proof of Theorem 1 is cast in the setting of hereditarily decomposable continua. In light of Bing's Theorem [1, Theorem 11, p. 660] that the properties atriodic and hereditarily unicoherent are characterizing properties for chainable continua among hereditarily decomposable continua, this proof applies only to the class of chainable continua.

\section{EXAMPLES}

Consider the one parameter family of mappings of $[0,1]$ given by $f_{\lambda}(x)=$ $4 \lambda x(1-x)$. For each positive integer $j$, there is a value for $\lambda$ such that $f_{\lambda}$ has a stable periodic orbit of period $2^{j}$. Then $M=\lim \left\{[0,1], f_{\lambda}\right\}$ is hereditarily decomposable and the shift homeomorphism on $M$ induced by $f$ has a periodic point whose period is $2^{j}$.

\section{REFERENCES}

1. R. H. Bing, Snake-like continua, Duke J. Math. 18 (1951), 653-663.

2. W. T. Ingram, Concerning periodic points in mappings of continua, to appear in Proc. Amer. Math. Soc., 104 (1988), 643-649.

3. D. P. Kuykendall, Irreducibility and indecomposability in inverse limits, Fund. Math. 84 (1973), 265-270.

4. P. Minc and W. R. R. Transue, Sarkovskii's Theorem for hereditarily decomposable chainable continua, preprint.

5. M. Smith and S. Young, Periodic homeomorphisms on T-like continua, Fund. Math. 104 (1979), 221-224.

6. R. H. Sorgenfrey, Concerning triodic continua, Amer. J. Math. 66 (1944), 439-460.

Department of Mathematics, University of Houston, Houston, Texas 77004

Current address: Department of Mathematics and Statistics, University of Missouri, Rolla, Missouri, 65401 